\title{
Bundesgesetz zum ePatientendossier: Der Aufwand hat sich gelohnt!
}

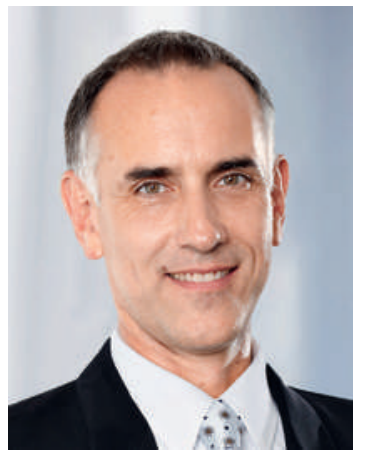

Am 29. Mai 2013 hat der Bundesrat die Botschaft zum Bundesgesetz über das elektronische Patientendossier (EPDG) an das Parlament überwiesen. Diesem Schritt ging ein mehrjähriger Prozess voraus.

Die Strategie eHealth Schweiz, die sich in die Handlungsfelder Elektronisches Patientendossier, Online-Dienste und Umsetzung Strategie eHealth aufteilt, wurde vom Bund zusammen mit den Kantonen erarbeitet und 2007 vom Bundesrat verabschiedet. Ziel war es, die bereits 1998 ausgearbeitete «Strategie für eine Informationsgesellschaft in der Schweiz» auf das Gesundheitswesen auszudehnen.

Bei der Umsetzung der nationalen eHealth-Strategie stellte sich schnell heraus, dass die Festlegung von Rahmenbedingungen, verbindlichen Regeln und einheitlichen Standards für die ganze Schweiz, über Kantons- und Institutionsgrenzen hinweg - und natürlich auch unabhängig von der Krankenversicherung - ein zentrales Anliegen ist. Dafür fehlte aber bisher nicht nur die gesetzliche Grundlage, auch die Verfassungsgrundlage ist eher dürftig.

Das EPDG soll nun einerseits die Anforderungen für eine sichere Bearbeitung von Gesundheitsdaten im elektronischen Patientendossier (EPD) regeln und andererseits die technischen Vorgaben festlegen, die einen standardisierten Datenaustausch auf nationaler Ebene ermöglichen. So hätten z.B.

1. Patienten Zugriff auf ihre medizinischen Daten und könnten entscheiden, wer diese einsehen darf und wer nicht. Zugleich stünden ihnen im Notfall alle wichtigen Daten zur Verfügung.

2. von Patienten berechtigte Ärzte einen Überblick und Einsicht in bereits erfolgte Behandlungen, erhobene Laborwerte und Medikationen des Patienten.

3. Spitäler die Möglichkeit, über die elektronische Austrittsdokumentation einerseits die zuweisenden Ärzte, andererseits aber auch die Pflegefachpersonen zeitgerecht zu involvieren, qualitätsrelevante Daten zu übermitteln und den Behandlungserfolg ihrer Eingriffe nachzuvollziehen.

Die FMH unterstützt die Festlegung einheitlicher Rahmenbedingungen für ein EPD auf nationaler Ebene. Sie hat den Entstehungsprozess der Vorlage eng begleitet. Die Arbeits- gruppe eHealth der FMH hat die verschiedenen Aspekte des Gesetzes und die mögliche Ausgestaltung intensiv diskutiert, teilweise auch direkt mit den Vertretern des BAG. Die FMH brachte ihre Positionen konsequent in Vernehmlassungen, Hearings und Arbeitsgruppen ein. Dieses Engagement hat sich gelohnt: Viele unserer Anliegen sind im nun vorliegenden Bundesgesetz aufgenommen. So hat man beispielsweise die Einwilligungsregelung praktikabler formuliert, ein $\mathrm{Zu}$ gang der Versicherer zu den Daten ist nicht vorgesehen, und das EPD bleibt sowohl für die Ärzte wie für die Patienten freiwillig. Ein von der AHV-Nummer unabhängiger Patientenidentifikator erhöht die Patientensicherheit und stärkt den Datenschutz. Last, but not least: Mit den vorgesehenen Finanzhilfen und der Absicht, den Aufwand für die Bearbeitung von Daten im EPD in den Tarifen zu berücksichtigen, könnte den Forderungen der FMH nach richtig zu setzenden Anreizen entsprochen werden.

\section{Zentrale Anliegen der Ärzteschaft sind im vorliegenden Bundesgesetz aufgenommen.}

Es ist unabdingbar, dass die Austauschbarkeit von Patientendaten unter verschiedenen Behandelnden - nur mit vorherigem Einverständnis der Patienten - über Institutions- und Kantonsgrenzen hinweg sichergestellt und gleichzeitig die Vertraulichkeit dieser Daten gewahrt sein muss. Die Sicherheit der Daten und die Selbstbestimmung des Patienten sind für die Akzeptanz entscheidende Erfolgsfaktoren. Das Patientendossier muss in den Behandlungsprozess integriert und die Behandelnden müssen in die Umsetzung eingebunden werden.

$\mathrm{Ob}$ aus diesem Gesetz ein für die Patientenbehandlung nützliches Werkzeug entsteht, wird entscheidend von der Ausgestaltung und damit auch von den Ausführungsbestimmungen abhängen. Auch hier wird sich die FMH erneut engagiert einbringen.

Dr. med. Gert Printzen, Mitglied des Zentralvorstandes der FMH, Verantwortlicher Ressort Medizinische Informatik und eHealth 